\title{
NitroSpeed-Carba NP Test for Rapid Detection and Differentiation between Different Classes of Carbapenemases in Enterobacterales
}

\author{
(D) Patrice Nordmann, a,b,c,d Mustafa Sadek, ${ }^{a}$ Anthony Demord, c Laurent Poirela,b,c \\ aMedical and Molecular Microbiology, Faculty of Science and Medicine, University of Fribourg, Fribourg, Switzerland \\ bINSERM European Unit (IAME), University of Fribourg, Fribourg, Switzerland \\ cSwiss National Reference Center for Emerging Antibiotic Resistance (NARA), University of Fribourg, Fribourg, Switzerland \\ IInstitute for Microbiology, University of Lausanne and University Hospital Centre, Lausanne, Switzerland
}

\begin{abstract}
A biochemical test (NitroSpeed-Carba NP test) was developed to identify carbapenemase production in Enterobacterales and to discriminate between the different types of clinically significant carbapenemases (Ambler classes A, B, and D). It is based on two main features, namely, the hydrolysis by all $\beta$-lactamases, including carbapenemases of the nitrocefin substrate, and the capacity of ertapenem to prevent this hydrolysis for all $\beta$-lactamases except carbapenemases. Specific carbapenemase inhibitors of class A (avibactam, vaborbactam), class B (dipicolinic acid), and class $D$ (avibactam) were used to inhibit the nitrocefin hydrolysis and to allow the identification of the carbapenemase types with a turnaround time of ca. $30 \mathrm{~min}$. The test was evaluated with a collection of 248 clinical enterobacterial isolates, including 148 carbapenemase producers and 100 non-carbapenemase producers. Its overall sensitivity and specificity were $100 \%$ and $97 \%$, respectively, including detection of all types of OXA-48-like carbapenemases. For the detection of the carbapenemase type, including strains that produce double carbapenemases, the sensitivity was $100 \%, 97 \%$, and $100 \%$ for the detection of classes A, B, and D, respectively. This easy-to-implement test may contribute to optimization of the choice of the $\beta$-lactam/ $\beta$-lactamase inhibitor combinations for treating infection due to carbapenemase producers.
\end{abstract}

KEYWORDS carbapenemase, Enterobacterales, diagnostics, rapid, Carba NP, UltraSpeed

reporte he growing incidence of multidrug resistance in Enterobacterales constitutes a major global health threat (1-3). Carbapenem resistance has been increasingly reported worldwide due to the increased use of carbapenems, which are supposed to be last-line agents retaining activity against multidrug-resistant Enterobacterales including those producing extended-spectrum $\beta$-lactamase (ESBL) and AmpC $\beta$-lactamases (4-6). Resistance to carbapenem among Enterobacterales is mediated by various mechanisms, including production of carbapenem-hydrolyzing enzymes (so-called carbapenemases), alteration in outer membrane permeability, and in certain circumstances, overproduction of an AmpC- or ESBL-type enzyme combined with porin loss/modification (7-10). The main clinically important carbapenemases identified in clinical isolates are grouped into three different classes according to their amino acid identity, corresponding to molecular class A (e.g., Klebsiella pneumoniae carbapenemase [KPC] enzymes), molecular class B (e.g., New Delhi metallo- $\beta$-lactamase [NDM], Verona integron-encoded metallo- $\beta$-lactamase [VIM], and imipenemase [IMP] enzymes), and class D (e.g., OXA-48 and its derivatives) $(9,11)$.
Citation Nordmann P, Sadek M, Demord A, Poirel L. 2020. NitroSpeed-Carba NP test for rapid detection and differentiation between different classes of carbapenemases in Enterobacterales. J Clin Microbiol 58:e00932-20. https://doi.org/10.1128/JCM.00932-20.

Editor Nathan A. Ledeboer, Medical College of Wisconsin

Copyright $\odot 2020$ American Society for Microbiology. All Rights Reserved.

Address correspondence to Patrice Nordmann, patrice.nordmann@unifr.ch.

Received 29 April 2020

Returned for modification 22 May 2020 Accepted 12 June 2020

Accepted manuscript posted online 24 June 2020

Published 24 August 2020

TThis article was published on 24 August 2020 with a CC BY 4.0 copyright line ("Copyright $\odot 2020$ Nordmann et al. This is an open-access article distributed under the terms of the Creative Commons Attribution 4.0 International license.") The authors elected to remove open access for the article after publication, necessitating replacement of the original copyright line, and this change was made on 29 September 2020.] 
Early and accurate detection of carbapenemase-producing organisms (CPOs) in infected or colonized patients is one of the most efficient ways to limit the dissemination of carbapenemase-producing isolates and also to optimally adapt the empirical treatment when needed. Most of the phenotype-based techniques not only suffer from some specificity and sensitivity issues but are also time-consuming (at least 12 to $24 \mathrm{~h}$ ) and lack guidance regarding the specific carbapenemase being produced; therefore, they are poorly adapted to the clinical need for isolating patients rapidly to prevent nosocomial outbreaks $(12,13)$. Molecular tests may be more rapid; however, they are often expensive, not easily implementable worldwide, and only detect the most common carbapenemase-encoding genes, missing the so-called "minor" or even unknown ones. The development of rapid carbapenemase detection tests such as the Carba NP test has represented a paradigm shift, since such a technique is not only rapid, specific, and sensitive but also implementable worldwide due to its low cost and ease of implementation. The Carba NP test is based on the biochemical detection of carbapenem (imipenem) hydrolysis $(12,14)$, meaning it may detect any kind of carbapenemase activity, regardless of its nature. Carbapenemase activity is evidenced through a color change (red to orange or yellow) of the buffer in which bacteria are tested, resulting from its acidification upon imipenem hydrolysis. However, this test suffers from the following two shortcomings: (i) its sensitivity is not optimal for some enzymes exhibiting weak carbapenemase activities, and (ii) it does not distinguish between different classes of carbapenemases.

Distinguishing between different classes of carbapenemases makes sense today, since novel $\beta$-lactamase inhibitors have been recently developed, counteracting, for instance, the activity of the class A carbapenemase KPC and/or class D carbapenemase OXA-48, although class B enzymes (metallo- $\beta$-lactamases [MBLs]) remain resistant to it. This is especially true for avibactam, which is a bridged diazabicyclo[3.2.1]octanone non- $\beta$-lactam inhibitor, inhibiting efficiently the aforementioned enzymes (15). This inhibitor is coupled with ceftazidime and soon will be with aztreonam, making excellent combinations that are active against KPC and OXA-48 producers but also MBL producers, respectively (16). Furthermore, the recently developed vaborbactam adds very significantly to the current armamentarium of $\beta$-lactamase inhibitors (17). Indeed, this first boronic acid inhibitor to be developed very efficiently inhibits classes $A$ and $D$ carbapenem-hydrolyzing $\beta$-lactamases but has no activity against OXA-48 and derivatives, while dipicolinic acid that is used only in vitro inhibits the activity of class B carbapenemases. Here, we developed a very rapid, simple, sensitive, and specific test for identification of carbapenemase-producing Enterobacterales, combining a capacity to assess which group the carbapenemase belongs to. The principle of this so-called NitroSpeed-Carba NP test relies, on one hand, on the hydrolysis of the nitrocefin substrate by all $\beta$-lactamases and, on the other hand, on the capacity of ertapenem (a carbapenem) to prevent this hydrolysis for all $\beta$-lactamases lacking carbapenemase activity. Indeed, enzymes lacking carbapenemase activity bind covalently and irreversibly with ertapenem and are no longer able to subsequently hydrolyze nitrocefin. By contrast, carbapenemases significantly hydrolyze ertapenem and are then still able to hydrolyze nitrocefin. This test was further developed by evaluating the capacity of different $\beta$-lactamase inhibitors (dipicolinic acid, avibactam, and vaborbactam) to inhibit the hydrolysis process of those carbapenemases.

\section{MATERIALS AND METHODS}

A genetically characterized collection of 248 various enterobacterial species from the Medical and Molecular Microbiology unit at the University of Fribourg, Switzerland, were included in this study. Isolates were obtained from various clinical sources (e.g., blood cultures, urine, sputum) and from various countries. The isolates included 144 carbapenemase producers and 100 non-carbapenemase producers. This collection included a large variety of enterobacterial species, including Escherichia coli, K. pneumoniae, Klebsiella oxytoca, Enterobacter cloacae, Enterobacter asburiae, Klebsiella aerogenes, Enterobacter hormaechei, Citrobacter freundii, Citrobacter braakii, Citrobacter murliniae, Citrobacter werkmanii, Providencia stuartii, Providencia rettgeri, Proteus mirabilis, Hafnia alvei, Morganella morganii, and Serratia marcescens. The carbapenemase types were as follows: class A carbapenemases ( $n=23$; KPC, NmcA, SME-1, GES, FRI, IMI), class B carbapenemases ( $n=66$; VIM, NDM, IMP, GIM), class D carbapenemases 
( $n=49$; OXA-48 and its derivatives). Notably, five isolates coproducing the NDM and OXA-48-like carbapenemases and a single isolate coproducing VIM and OXA-48-like enzymes were included (Table 1). In addition, four isolates producing enzymes that are not detected by using the Carba NP test (namely, CTX-M-33, SHV-38, and overexpressed OXY $\beta$-lactamase from $K$. oxytoca) were also tested. Previous studies reported that those four enzymes are exhibiting a very low level of carbapenemase activity; therefore, we wanted to check if our test is able to detect such a low level of carbapenemase activity (18-20). Quality control (QC) strains have been included with well-characterized carbapenemases. They included two E. coli strains producing NDM-5 and OXA-48-like carbapenemases (strains N679 and N1084, respectively) and two K. pneumoniae strains producing KPC-2 and NDM-1 (strains R307 and N1086, respectively). The $\beta$-lactamase gene content of those four isolates was determined by whole-genome sequencing. One hundred non-carbapenemase producers were tested that included ESBL and non-ESBL $(n=50)$ producers of plasmid-mediated AmpC enzymes, and isolates combining production of their chromosomally encoded AmpC together with a decreased membrane permeability $(n=49)$ were also included as well as a single $\beta$-lactamase-negative E. coli reference strain ATCC 25922 (Table 1). The antimicrobial resistance profiles of the strains were determined by disk diffusion according to EUCAST (http://www.eucast.org/clinical_breakpoints/) guidelines. The MICs of ertapenem, meropenem, and imipenem was determined using Etest strips (bioMérieux, La Balme-les-Grottes, France) on Mueller-Hinton agar plates at $37^{\circ} \mathrm{C}$. Etest (including QC) was performed according to the manufacturer's instructions, and results were interpreted according to the latest EUCAST breakpoints (Table 1). All strains had previously been characterized for their $\beta$-lactamase content and carbapenemase-encoding genes by PCR approaches followed by subsequent DNA sequencing.

The NitroSpeed-Carba NP test. The test was prepared and performed as follows (Fig. 1). For each bacterial isolate, 1 to 2 calibrated $1-\mu$ l loopfuls of tested colonies, grown overnight at $37^{\circ} \mathrm{C}$ on UriSelect 4 (Bio-Rad, Marnes-la-Coquette, France; reference no. 64694), Mueller-Hinton agar (Bio-Rad; reference no. 64884), or Luria-Bertani agar (Carl Roth GmbH and Co., Karlsruhe; article no. X969.2) plates or primary specimen plating (e.g., blood agar plates) were selected. To extract the enzymes, the sample was resuspended in one of the following: (i) $100 \mu \mathrm{l}$ of a Tris-HCl $20 \mathrm{mmol} /$ liter lysis buffer (B-PER II bacterial protein extraction reagent; Thermo Scientific Pierce, Rockford, IL, USA) containing $0.1 \mathrm{mM} \mathrm{ZnSO}_{4}$ (Carl Roth $\mathrm{GmbH}$; reference no. $\mathrm{K} 301.1$ ), $\mathrm{pH}$ 7.5, in tubes 1 and 2; (ii) $100 \mu \mathrm{l}$ of a Tris- $\mathrm{HCl} 20 \mathrm{mmol} /$ liter lysis buffer containing $0.1 \mathrm{mM} \mathrm{ZnSO}_{4}$ and $25 \mathrm{mM}$ dipicolinic acid (Sigma Aldrich; reference no. P63808), pH 7.5, in tube 3; (iii) $100 \mu \mathrm{l}$ of a Tris- $\mathrm{HCl} 20 \mathrm{mmol} /$ liter lysis buffer containing $0.1 \mathrm{mM} \mathrm{ZnSO}_{4}$ and avibactam sodium hydrate $(0.04 \mathrm{mM}[12.2 \mu \mathrm{g} / \mathrm{ml}]$; MedChemExpress; reference no. HY-14879B) in tube 4; or (iv) $100 \mu \mathrm{l}$ of a Tris-HCl $20 \mathrm{mmol} /$ liter lysis buffer containing $0.1 \mathrm{mM} \mathrm{ZnSO}_{4}$ and vaborbactam $(50 \mu \mathrm{g} / \mathrm{ml}$; MedChemExpress; reference no. HY-19930) in tube 5.

Tubes were then vortexed vigorously for 30 to $60 \mathrm{~s}$ until complete resuspension. Then, $50 \mu \mathrm{l}$ of distilled water was added to tube 1 (control) and $50 \mu \mathrm{l}$ of ertapenem sodium (ETP) ( $80 \mu \mathrm{g} / \mathrm{ml}$; SigmaAldrich; reference no. SML1238) to tubes 2 to 5 . Following incubation at room temperature for $5 \mathrm{~min}$, $50 \mu \mathrm{l}$ of $1 \mathrm{~g} /$ /iter nitrocefin (Toronto Research Chemicals, Canada; reference no. N493815) was added into each tube 1 to 5 . The final concentrations of ETP were $20 \mu \mathrm{g} / \mathrm{ml}$.

The test was considered as interpretable if one of the following 5 conditions was met (Fig. 1). (i) A color change from yellow to red (hydrolysis of nitrocefin) in tubes 1,2, and 3 and the absence of any color change (no hydrolysis of nitrocefin) in tube 4 containing ertapenem plus avibactam and tube 5 containing ertapenem plus vaborbactam indicate class A carbapenemase production (e.g., KPC). (ii) A color change from yellow to red (hydrolysis of nitrocefin) in tubes 1, 2, 4, and 5 and the absence of any color change (no hydrolysis of nitrocefin) in tube 3 containing ertapenem and dipicolinic acid indicate production of a class B carbapenemase, such as NDM, VIM, IMP, or GIM. (iii) A color change from yellow to red (hydrolysis of nitrocefin) in tubes 1, 2, 3, and 5 and the absence of any color change (no hydrolysis of nitrocefin) in tube 4 containing ertapenem and avibactam indicate production of a class D carbapenemase, such as OXA-48 and its derivatives. (iv) A color change from yellow to red in tube 1 only containing water and the absence of any color change (no hydrolysis of nitrocefin) in the other tubes 2 to 5 indicate lack of carbapenemase activity but production of a noncarbapenemase $\beta$-lactamase, such as TEM-1 or CTX-M-type enzymes. (v) The absence of any color change (no hydrolysis of nitrocefin) in all tubes (1 to 5 ) indicates lack of $\beta$-lactamase activity.

All results are interpreted within $15 \mathrm{~min}$ ( $10 \mathrm{~s}$ to $15 \mathrm{~min}$ ) of incubation at room temperature. The different readers of the tests were blinded from the type of isolates tested.

\section{RESULTS AND DISCUSSION}

A rapid, sensitive, and specific test has been developed here, not only for ultrarapid and simple detection of carbapenemase-producing Enterobacterales but also to identify the different classes of carbapenemases. This test is a derivative of the Nitro-Carba test (21) but presents here the significant advantage of identifying the carbapenemases according to the classes they belong to. The NitroSpeed-Carba NP test allows rapid identification of all types of carbapenemase producers, including isolates producing KPC, OXA-48-types, NDM, VIM, and IMP, with a turnaround time of ca. 30 min (only 10 to $15 \mathrm{~min}$ for the final reaction after the addition of nitrocefin). The formerly developed Carba NP test allowed rapid identification of carbapenemase production by detecting acid products resulting from the hydrolysis of imipenem in the presence of a phenol 
TABLE 1 Features of clinical enterobacterial isolates, MICs of carbapenems, and results of the NitroSpeed-Carba NP test ${ }^{a}$

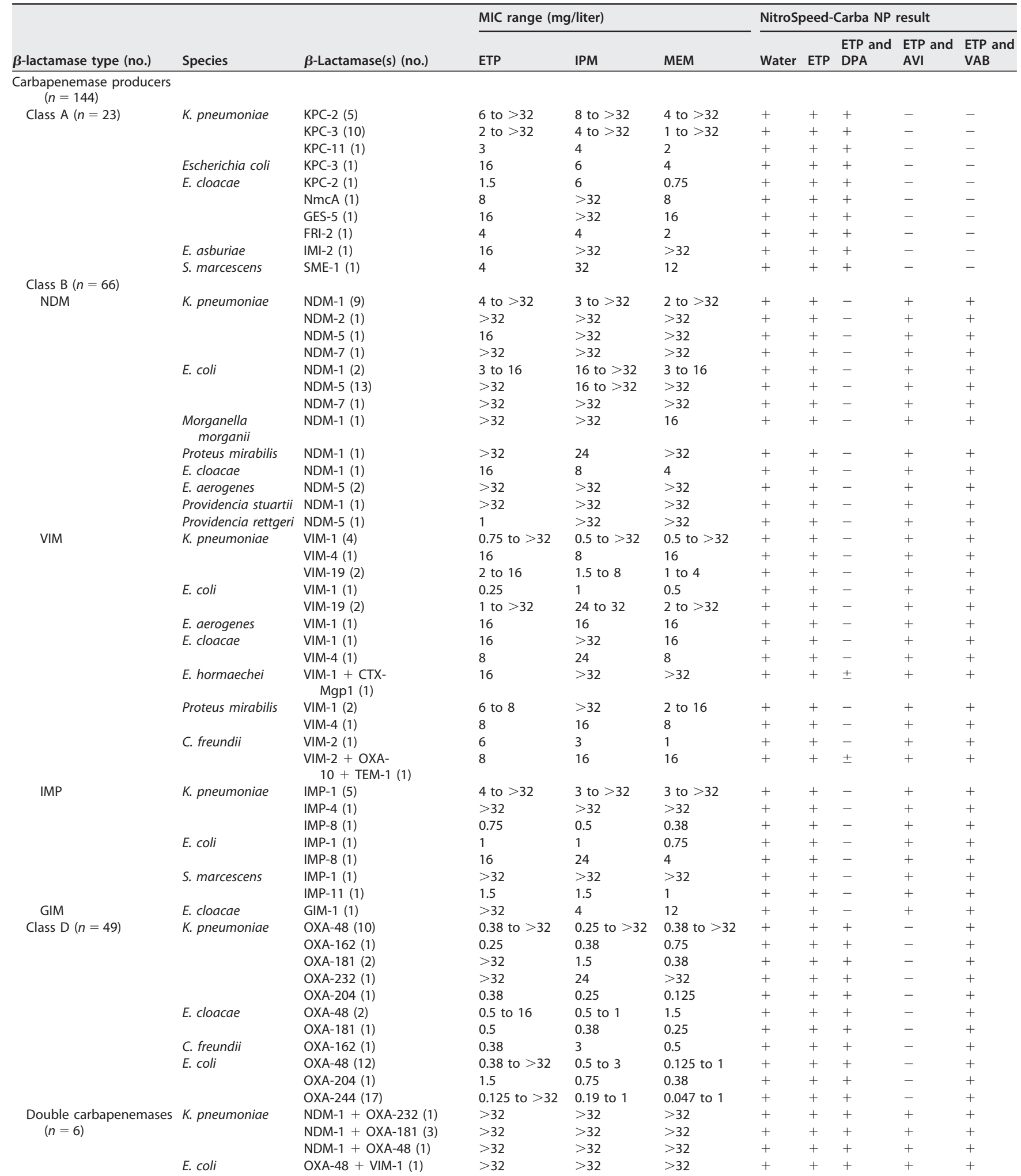


TABLE 1 (Continued)

\begin{tabular}{|c|c|c|c|c|c|c|c|c|c|c|}
\hline \multirow[b]{2}{*}{$\boldsymbol{\beta}$-lactamase type (no.) } & \multirow[b]{2}{*}{ Species } & \multirow[b]{2}{*}{$\boldsymbol{\beta}$-Lactamase(s) (no.) } & \multicolumn{3}{|c|}{ MIC range (mg/liter) } & \multicolumn{5}{|c|}{ NitroSpeed-Carba NP result } \\
\hline & & & ETP & IPM & MEM & Water & ETP & $\begin{array}{l}\text { ETP and } \\
\text { DPA }\end{array}$ & $\begin{array}{l}\text { ETP and } \\
\text { AVI }\end{array}$ & $\begin{array}{l}\text { ETP and } \\
\text { VAB }\end{array}$ \\
\hline \multirow{19}{*}{$\begin{array}{l}\text { ESBL producers } \\
(n=50)\end{array}$} & \multirow[t]{4}{*}{ E. coli } & OXA-1 (5) & 0.003 to 0.125 & 0.19 to 0.25 & 0.016 to 0.023 & + & - & - & - & - \\
\hline & & TEM (3) & 0.032 & 0.125 & 0.047 & + & - & - & - & - \\
\hline & & CTX-M-15 (8) & 1.5 to $>32$ & 0.38 to 1 & 0.75 to 0.19 & + & - & - & - & - \\
\hline & & CTX-M-3 (2) & 0.125 & 0.25 & 0.125 & + & - & - & - & - \\
\hline & \multirow[t]{4}{*}{ E. cloacae } & TEM-3 (2) & 0.125 & 0.125 & 0.125 & + & - & - & - & - \\
\hline & & CTX-M-3 + TEM-1 (1) & 0.38 & 0.125 & 0.25 & + & - & - & - & - \\
\hline & & CTX-M-3 (1) & 0.38 & 0.12 & 0.25 & + & - & - & - & - \\
\hline & & OXA-163 (1) & 2 & 0.5 & 0.5 & + & - & - & - & - \\
\hline & E. aerogenes & CTX-M-3 (2) & 0.125 & 0.125 & 0.125 & + & - & - & - & - \\
\hline & \multirow[t]{6}{*}{ K. pneumoniae } & CTX-M-3 (2) & 0.125 & 0.38 & 0.125 & + & - & - & - & - \\
\hline & & CTX-M-15 (9) & 0.064 to 16 & 0.25 to 8 & 0.75 to 32 & + & - & - & - & - \\
\hline & & SHV-5 (2) & 0.125 & 0.125 & 0.125 & + & - & - & - & - \\
\hline & & SHV-11 (1) & 0.047 & 0.19 & 0.023 & + & - & - & - & - \\
\hline & & CTX-M-1 (3) & 0.125 to 0.25 & 0.125 to 0.25 & 0.12 to 0.25 & + & - & - & - & - \\
\hline & & CTX-M-3 + TEM-1 (2) & 0.38 & 0.125 & 0.38 & + & - & - & - & - \\
\hline & K. oxytoca & CTX-M-3 + TEM-1 (1) & 0.125 & 0.125 & 0.38 & + & - & - & - & - \\
\hline & \multirow[t]{3}{*}{ C. freundii } & CTX-M-1 (1) & 0.047 & 0.25 & 0.016 & + & - & - & - & - \\
\hline & & CTX-M-3 (3) & 0.047 & 0.38 to 2 & 0.032 to 0.047 & + & - & - & - & - \\
\hline & & CTX-M-15 + TEM-1 (1) & 0.38 & 0.25 & 0.19 & + & - & - & - & - \\
\hline \multirow{24}{*}{$\begin{array}{l}\text { Non-ESBL producers } \\
\quad(n=49)\end{array}$} & K. pneumoniae & DHA (10) & 0.75 to $>32$ & 0.5 to $>32$ & 0.19 to $>32$ & + & - & - & - & - \\
\hline & \multirow[t]{5}{*}{ E. coli } & CMY + CTX-M-15 (1) & 0.125 & 0.125 & 0.25 & + & - & - & - & - \\
\hline & & CMY-2 like (1) & 0.094 & 0.38 & 0.064 & + & - & - & - & - \\
\hline & & CMY + LAT (1) & 0.5 & 0.5 & 0.125 & + & - & - & - & - \\
\hline & & CMY (1) & 0.38 & 0.125 & 0.125 & + & + & - & - & - \\
\hline & & $A C C-1(1)$ & 0.125 & 0.38 & 0.125 & + & + & - & - & - \\
\hline & Proteus mirabilis & CMY (1) & 0.064 & 3 & 0.125 & + & + & - & - & - \\
\hline & \multirow[t]{2}{*}{ Hafnia alvei } & ACC-2 (4) & 0.032 to 0.38 & 0.38 to 0.5 & 0.047 to 0.094 & + & - & - & - & - \\
\hline & & $\begin{array}{l}\text { Overexpressed AmpC } \\
\text { cephalosporinase (1) }\end{array}$ & 12 & 0.25 & 1 & + & - & - & - & - \\
\hline & \multirow[t]{3}{*}{ C. freundii } & WT (3) & 0.012 to 0.064 & 0.38 & 0.032 to 0.047 & + & - & - & - & - \\
\hline & & AmpC cephalosporinase (2) & 0.094 to 12 & 0.38 to 2 & 0.38 to 6 & + & - & - & - & - \\
\hline & & ACC-2 (1) & 0.008 & 0.19 & 0.032 & + & - & - & - & - \\
\hline & \multirow[t]{2}{*}{ C. braakii } & WT (1) & 0.016 & 0.38 & 0.064 & + & - & - & - & - \\
\hline & & AmpC cephalosporinase (2) & 0.006 & 0.25 & 0.016 & + & - & - & - & - \\
\hline & C. murliniae & AmpC cephalosporinase (1) & 0.006 & 0.25 & 0.016 & + & - & - & - & - \\
\hline & C. werkmanii & AmpC cephalosporinase (1) & 0.008 & 0.05 & 0.023 & + & - & - & - & - \\
\hline & \multirow{3}{*}{$\begin{array}{l}\text { Morganella } \\
\text { morganii }\end{array}$} & WT (1) & 0.023 & 1.5 & 0.125 & + & - & - & - & - \\
\hline & & Overexpressed AmpC (1) & 0.64 & 32 & 0.25 & + & - & - & - & - \\
\hline & & $\begin{array}{l}\text { Natural cephalosporinase } \\
\text { (1) }\end{array}$ & 0.23 & 1.5 & 0.125 & + & - & - & - & - \\
\hline & E. aerogenes & $\begin{array}{l}\text { Overexpressed AmpC } \\
\text { cephalosporinase (1) }\end{array}$ & 32 & 16 & 4 & + & - & - & - & - \\
\hline & \multirow[t]{4}{*}{ E. cloacae } & WT (1) & 0.012 & 0.064 & 0.12 & + & - & - & - & - \\
\hline & & $\begin{array}{l}\text { Overexpressed AmpC } \\
\text { cephalosporinase (8) }\end{array}$ & 0.38 to $>32$ & 0.38 to 2 & 0.094 to 4 & + & - & - & - & - \\
\hline & & $\begin{array}{l}\text { Natural cephalosporinase } \\
\text { (2) }\end{array}$ & 0.125 & 0.38 & 0.12 & + & - & - & - & - \\
\hline & & CMY (2) & 1 to $>32$ & 0.38 to $>32$ & 0.125 to $>32$ & + & - & - & - & - \\
\hline Negative control $(n=1)$ & E. coli & ATCC 25922 (1) & 0.003 & 0.125 & 0.008 & - & - & - & - & - \\
\hline
\end{tabular}

aWT, wild type; ETP, ertapenem; IPM, Imipenem; MEM, meropenem; DPA, dipicolinic acid; AVI, avibactam; VAB, vaborbactam; +, red; -, yellow; \pm , reddish yellow.

red as a $\mathrm{pH}$ indicator (12). Although the Carba NP test requires an additional 30-min extraction step, the NitroSpeed-Carba NP is performed by the direct use of a bacterial cell suspension in the lysis solution without an incubation period. The detection of some OXA-type carbapenemases by using the Carba NP test may be challenging due to (i) their weak carbapenemase activity, (ii) the low level of expression of the corresponding genes, and (iii) in some cases, a limited copy number of the corresponding gene (e.g., a single copy of the carbapenemase gene may be inserted into the chromosome). Therefore, PCR-based methods often remain useful for the identification of those carbapenem-hydrolyzing class D $\beta$-lactamases (22). However, these methods usually require an additional step of bacterial culture (additional $24 \mathrm{~h}$ ) and then 3 to $8 \mathrm{~h}$ to be performed and require specific, expensive equipment and a significant degree of expertise, apart from the high costs associated.

For the detection of carbapenemase production, the sensitivity and specificity of the NitroSpeed-Carba NP test were 100\% (144/144) and 97\% (97/100), respectively. Ertap- 


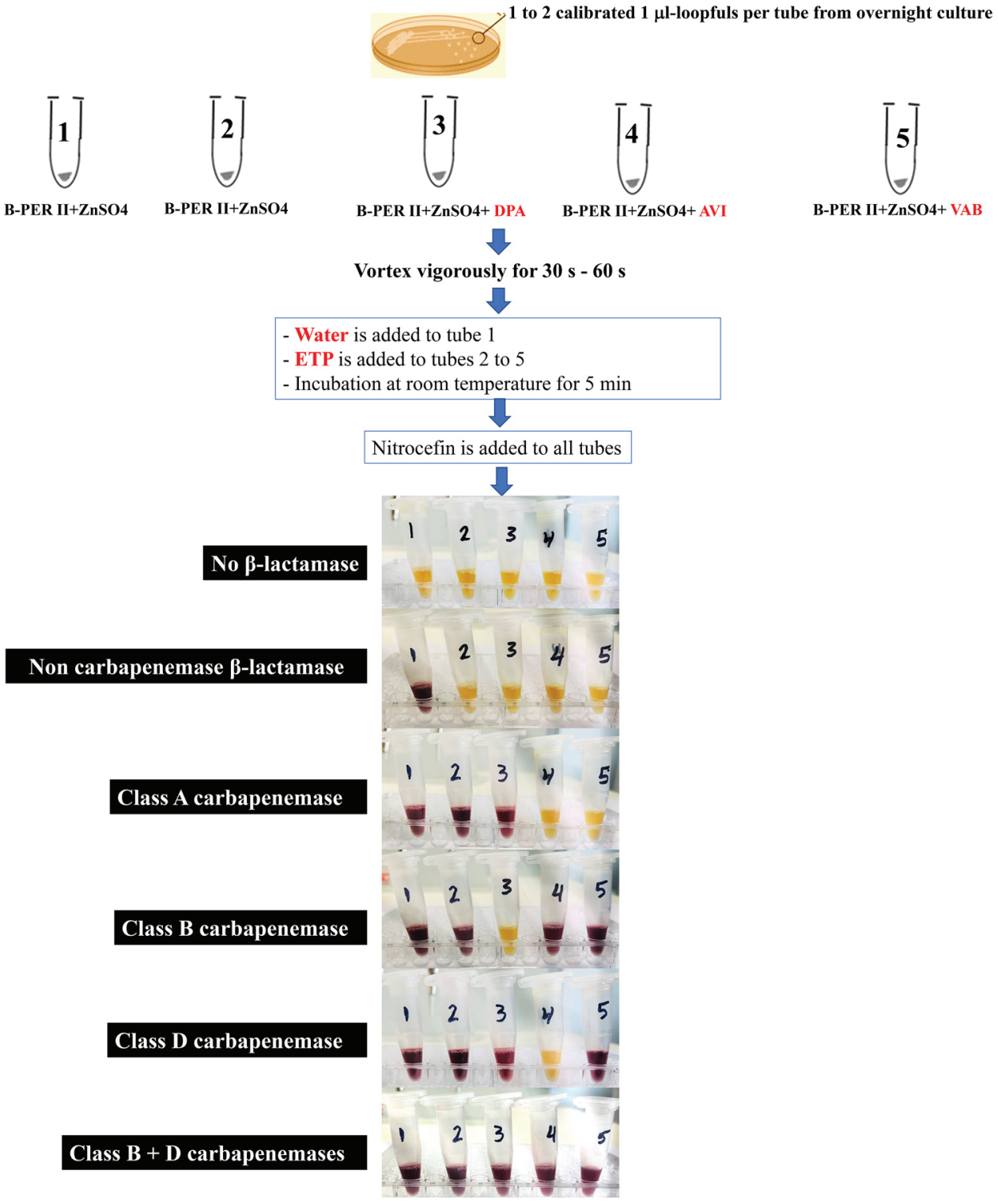

FIG 1 Detailed protocol of the NitroSpeed-Carba NP test.

enem was found to prevent nitrocefin hydrolysis by ESBLs, AmpC $\beta$-lactamases, and coproduced ESBL and AmpC $\beta$-lactamases (negativity of the NitroSpeed-Carba NP test), therefore, permitting the differentiation between carbapenem-resistant Enterobacterales producing or not producing carbapenemases. The only three strains in our collection that gave false-positive results were producers of ACC- or CMY-type AmpC enzymes that are known to have very slight carbapenemase activity. The NitroSpeed-Carba NP test showed excellent results for detecting any OXA-48-type producer, including the difficult-to-detect OXA-244 producers $(23,24)$. The NitroSpeed-Carba NP test allowed categorization of the different carbapenemase classes. The sensitivity of the NitroSpeed-Carba NP test was 100\% (23/23), 97\% (64/66), and 100\% (49/49) for the detection of class A, B, and D carbapenemases, respectively. Those values were obtained even by including strains that produced two types of unrelated carbapenemases, for which the precise identification of the carbapenemase is not possible. Noteworthy, the test could detect producers of two carbapenemases, such as those producing NDM and OXA-48-type or VIM and OXA-48-type enzymes (sensitivity, 100\% $[6 / 6])$. In addition, the NitroSpeed-Carba NP test was positive for strains producing 
enzymes with very-low-level carbapenemase activity (sensitivity, 100\% [4/4]), such as CTX-M-33 (18), SHV-38 (19), and overexpressed $\beta$-lactamase OXY in $K$. oxytoca, with corresponding carbapenemase activities not detected using the Carba NP test (20). Detection of all carbapenemase producers was possible using the NitroSpeed-Carba NP test, regardless of the respective levels of carbapenemase activity. It was observed that the positivity of the NitroCarba NP test was immediate (less than $1 \mathrm{~min}$ ) when testing enzymes with high carbapenemase activity (such as KPC-2), whereas it was a bit delayed (for instance, 15-min incubation) for enzymes showing low-level carbapenemase activity (such as CTX-M-33). Moreover, the NitroSpeed-Carba NP test gave a negative result when testing an OXA-163 producer, that latter OXA-48-like $\beta$-lactamase possessing a unique hydrolytic profile mirroring that of class A ESBLs but almost lacking any carbapenemase activity (25).

The NitroSpeed-Carba NP test will be further compared to the $\beta$-Carba test, which is known to be less sensitive than the Carba NP test for detecting carbapenemase activity (14). The rapid immunochromatography-based detection of carbapenemase producers is another technique that can detect carbapenemases (KPC, IMP, VIM, OXA-48 like enzymes) (26). However, that latter technique cannot detect several carbapenemases, such as GES, NMC-A, SME, FRI, or IMI enzymes, whereas the NitroSpeed-Carba NP can detect any type of carbapenemase including some potentially novel ones.

In conclusion, the NitroSpeed-Carba NP test is an easy-to-perform and reliable test for rapid identification of carbapenemase-producing Enterobacterales, with excellent sensitivity and specificity. Use of this test that additionally presents the advantage to rapidly identify the carbapenemase type does not require any specific equipment. Given its user-friendliness, simplicity, and short turnaround time to result, the NitroSpeed-Carba NP test would be suitable for any microbiology laboratory, in particular if a commercial test is developed.

Finally, use of this test may contribute to the choice of $\beta$-lactamase/ $\beta$-lactamase inhibitor combinations for treating infections due to carbapenemase producers. Indeed, ceftazidime-avibactam may be proposed for treating infections due to KPC or OXA-48 producers but not due to metallo-carbapenemase producers, whereas the spectrum of activity of meropenem-vaborbactam includes KPC producers only. Finally, since resistance to ceftazidime-avibactam and meropenem-vaborbactam may occur among Enterobacterales (16), the NitroSpeed-Carba NP test may provide the opportunity to easily monitor the occurrence of those multidrug-resistant isolates.

\section{ACKNOWLEDGMENT}

This work was funded by the Swiss National Science Foundation (project numbers FNS-407240_177381 and FNS-407240_177382).

\section{REFERENCES}

1. Schwaber MJ, Carmeli Y. 2008. Carbapenem-resistant Enterobacteriaceae: a potential threat. JAMA 300:2911-2913. https://doi.org/10.1001/jama .2008 .896$.

2. Pitout JD, Laupland KB. 2008. Extended-spectrum $\beta$-lactamase-producing Enterobacteriaceae: an emerging public-health concern. Lancet Infect Dis 8:159-166. https://doi.org/10.1016/S1473-3099(08)70041-0.

3. Infectious Diseases Society of America (IDSA), Spellberg B, Blaser $M$, Guidos RJ, Boucher HW, Bradley JS, Eisenstein BI, Gerding D, Lynfield R, Reller LB, Rex J, Schwartz D, Septimus E, Tenover FC, Gilbert DN. 2011. Combating antimicrobial resistance: policy recommendations to save lives. Clin Infect Dis 52:S397-S428. https://doi.org/10.1093/cid/cir153.

4. Perez F, Endimiani A, Hujer KM, Bonomo RA. 2007. The continuing challenge of ESBLs. Curr Opin Pharmacol 7:459-469. https://doi.org/10 .1016/j.coph.2007.08.003.

5. Jacoby GA. 2009. AmpC $\beta$-lactamases. Clin Microbiol Rev 22:161-182. https://doi.org/10.1128/CMR.00036-08.

6. Tängdén T, Giske CG. 2015. Global dissemination of extensively drugresistant carbapenemase-producing Enterobacteriaceae: clinical perspec- tives on detection, treatment and infection control. J Intern Med 277: 501-512. https://doi.org/10.1111/joim.12342.

7. Yang D, Guo Y, Zhang Z. 2009. Combined porin loss and extended spectrum $\beta$-lactamase production is associated with an increasing imipenem minimal inhibitory concentration in clinical Klebsiella pneumoniae strains. Curr Microbiol 58:366-370. https://doi.org/10.1007/ s00284-009-9364-4.

8. Thomson KS. 2010. Extended-spectrum- $\beta$-lactamase, AmpC, and carbapenemase issues. J Clin Microbiol 48:1019-1025. https://doi.org/10.1128/ JCM.00219-10.

9. Nordmann P, Naas T, Poirel L. 2011. Global spread of carbapenemaseproducing Enterobacteriaceae. Emerg Infect Dis 17:1791-1798. https:// doi.org/10.3201/eid1710.110655.

10. Papp-Wallace KM, Endimiani A, Taracila MA, Bonomo RA. 2011. Carbapenems: past, present, and future. Antimicrob Agents Chemother 55:4943-4960. https://doi.org/10.1128/AAC.00296-11.

11. Jean SS, Lee WS, Lam C, Hsu CW, Chen RJ, Hsueh PR. 2015. Carbapenemaseproducing Gram-negative bacteria: current epidemics, antimicrobial sus- 
ceptibility and treatment options. Future Microbiol 10:407-425. https://doi .org/10.2217/fmb.14.135.

12. Nordmann P, Poirel L, Dortet L. 2012. Rapid detection of carbapenemaseproducing Enterobacteriaceae. Emerg Infect Dis 18:1503-1507. https://doi .org/10.3201/eid1809.120355.

13. Tamma PD, Simner PJ. 2018. Phenotypic detection of carbapenemaseproducing organisms from clinical isolates. J Clin Microbiol 56:e01140 -18. https://doi.org/10.1128/JCM.01140-18.

14. Mancini S, Kieffer N, Poirel L, Nordmann P. 2017. Evaluation of the Rapidec Carba NP and $\beta$-Carba tests for rapid detection of carbapenemaseproducing Enterobacteriaceae. Diagn Microbiol Infect Dis 88:293-297. https://doi.org/10.1016/j.diagmicrobio.2017.05.006.

15. Drawz SM, Papp-Wallace KM, Bonomo RA. 2014. New $\beta$-lactamase inhibitors: a therapeutic renaissance in an MDR world. Antimicrob Agents Chemother 58:1835-1846. https://doi.org/10.1128/AAC.00826-13.

16. Ackley R, Roshdy D, Meredith J, Minor S, Anderson WE, Capraro GA, Polk C. 2020. Meropenem-vaborbactam versus ceftazidime-avibactam for treatment of carbapenem-resistant Enterobacteriaceae infections. Antimicrob Agents Chemother 64:e02313-19. https://doi.org/10.1128/AAC .02313-19.

17. Hecker SJ, Reddy KR, Totrov M, Hirst GC, Lomovskaya O, Griffith DC, King P, Tsivkovski R, Sun D, Sabet M, Tarazi Z, Clifton MC, Atkins K, Raymond A, Potts KT, Abendroth J, Boyer SH, Loutit JS, Morgan EE, Durso S, Dudley MN. 2015. Discovery of a cyclic boronic acid beta-lactamase inhibitor (RPX7009) with utility vs class A serine carbapenemases. J Med Chem 58:3682-3692. https://doi.org/10.1021/acs.jmedchem.5b00127.

18. Poirel L, Ortiz de la Rosa JM, Richard A, Aires-de-Sousa M, Nordmann P. 2019. CTX-M-33 is a CTX-M-15 derivative conferring reduced susceptibility to carbapenems. Antimicrob Agents Chemother 63:e01515-19. https://doi.org/10.1128/AAC.01515-19.

19. Poirel L, Héritier C, Podglajen I, Sougakoff W, Gutmann L, Nordmann P. 2003. Emergence in Klebsiella pneumoniae of a chromosome-encoded
SHV $\beta$-lactamase that compromises the efficacy of imipenem. Antimicrob Agents Chemother 47:755-758. https://doi.org/10.1128/aac.47.2 .755-758.2003.

20. Yang S, Hemarajata P, Shevy L, Maciariello M, Culbreath K, Bush K, Humphries R. 2018. Unusual carbapenem resistant but ceftriaxone and cefepime susceptible Klebsiella oxytoca isolated from a blood culture: case report and whole-genome sequencing investigation. IDCases 11 : 9-11. https://doi.org/10.1016/j.idcr.2017.11.007.

21. Teethaisong Y, Nakouti I, Evans K, Eumkeb G, Hobbs G. 2019. Nitro-Carba test, a novel and simple chromogenic phenotypic method for rapid screening of carbapenemase-producing Enterobacteriaceae. J Glob Antimicrob Resist 18:22-25. https://doi.org/10.1016/j.jgar.2019.01.012.

22. Poirel L, Naas T, Nordmann P. 2010. Diversity, epidemiology, and genetics of class $D \beta$-lactamases. Antimicrob Agents Chemother 54:24-38. https://doi.org/10.1128/AAC.01512-08.

23. Potron A, Poirel L, Dortet L, Nordmann P. 2016. Characterisation of OXA-244, a chromosomally-encoded OXA-48-like $\beta$-lactamase from Escherichia coli. Int J Antimicrob Agents 47:102-103. https://doi.org/10 .1016/j.ijantimicag.2015.10.015.

24. Hoyos-Mallecot $Y$, Naas T, Bonnin RA, Patino R, Glaser P, Fortineau N, Dortet L. 2017. OXA-244-producing Escherichia coli isolates, a challenge for clinical microbiology laboratories. Antimicrob Agents Chemother 61:e00818-17. https://doi.org/10.1128/AAC.00818-17.

25. Poirel L, Castanheira M, Carrër A, Rodriguez CP, Jones RN, Smayevsky J, Nordmann P. 2011. OXA-163, an OXA-48-related class D $\beta$-lactamase with extended activity toward expanded-spectrum cephalosporins. Antimicrob Agents Chemother 55:2546-2551. https://doi.org/10.1128/AAC .00022-11.

26. Kieffer N, Poirel L, Nordmann P. 2019. Rapid immunochromatographybased detection of carbapenemase producers. Infection 47:673-675. https://doi.org/10.1007/s15010-019-01326-1. 